\title{
Comunidades científicas e infraestrutura tecnológica no Brasil para uso de recursos eletrônicos de comunicação e informação na pesquisa
}

\section{Lena Vania Ribeiro Pinheiro}

Instituto Brasileiro de Informação em Ciência e Tecnologia-IBICT/ Coordenação de Ensino e Pesquisa. Doutora em Comunicação e Cultura, UFRJ/ECO.

E-mail: Ienavania@montreal.com.br

\section{Resumo}

Estudo do uso de recursos eletrônicos de comunicação e informação por pesquisadores brasileiros, na geração de novos conhecimentos. A análise abrange a freqüencia de participação na rede, fatores intervenientes, finalidades de uso e o grau de relevância dessa tecnologia. No acesso e uso da informação científica e técnica, são comparados os recursos eletrônicos/ digitais e os impressos/ tradicionais. A partir dos resultados, são identificadas percepções e perspectivas, entre as quais a aproximação entre comunicação científica e divulgação científica, a confluência dos processos de comunicação e informação e o crescente interesse pela temática da pesquisa, inclusive no Brasil. Pela importância dos recursos eletrônicos para pesquisa, há necessidade de sua expansão e atualização constantes, com investimento das instituições e sua inserção nas políticas públicas nacionais.

\section{Palavras-chave}

Comunicação científica no Brasil; Recursos eletrônicos de comunicação; Recursos eletrônicos de informação; Comunidades científicas brasileiras; Uso de tecnologias de informação.

\section{Scientific communities and technological} infrastructure in Brazil for use of electronic resources of communication and information in research

\section{Abstract \\ Usage study of communication and information electronic resources by Brazilian researchers in the generation of new knowledge. The analysis encompasses the frequency of participation in the network as well as the intervenient factors, usage aims and the relevance of this technology. Electronic resources and traditional printed ones are compared in scientific and technical information usage and access. Results identified perceptions and perspectives, among which the coming together of scientific communication and scientific divulgation, the confluence of communication and information processes and the growing interest for research in the area, including Brazil. \\ Considering the importance of electronic resources for research, there is a need for its constant expansion and updating, which implies investments by Brazilian institutions and its insertion in the national public policies. \\ Keywords}

Scientific communication in Brazil; Electronic resources of communication; Electronic resources of information; Brazilian Scientific communities; Use of information technologies.

\section{IN T RO D U ÇÃ O}

0 projeto integrado de pesquisa "Impactos das redes eletrônicas na comunicação científica e novos territórios cognitivos para práticas coletivas, interativas e interdisciplinares", financiado pelo Conselho $\mathrm{N}$ acional de Desenvolvimento Científico e Tecnológico (CN Pq) e desenvolvido durante quatro anos (1998-2002), teve objetivos mais amplos, como o próprio título traduz, abrangendo as repercussões dessas tecnologias na produção de pesquisas e geração de conhecimento, em termos de comunicação científica, expansão das equipes, intensificação da comunicação e relações interdisciplinares, nos múltiplos aspectos da questão (Pinheiro, coord. 2002)*.

0 presente artigo restringe-se ao uso dos recursos eletrônicos de comunicação e informação. No primeiro caso, destacamos o correio eletrônico, as listas de discussão, as salas virtuais (chats). No segundo, as bibliotecas virtuais e digitais, entre outros.

Estudos de comunicação científica naturalmente se estendem até a informação científica e tecnológica, ou vice-versa, por uma razão principal: a informação científica e tecnológica é parte fundamental da infraestrutura de $C \&$ T. Portanto, abordar a comunicação científica significa não somente enfocar padrões de comunicação entre pares, mas também englobar tanto a informação à qual recorrem para as suas pesquisas quanto aquela que produzem e transmitem por diferentes canais de comunicação e tipos de documentos. Comunicação e informação científicas estão, ainda, estreitamente relacionadas às políticas de $C \& T$ (Pinheiro, 2000)

\footnotetext{
* Participaram deste projeto, entre outros, na ocasião mestrandos e doutorandos do Programa de Pós-Graduação em Ciência da Informação (PPGCI), da linha de pesquisa Tecnologia de Informação, desenvolvida no Ibict, os doutores Sandra Rebel Gomes e mestres Miguel Angel Schwindt, Michell S. da Costa e Ângela Cardoso Braga, além da bolsista de apoio técnico Lea Regina de Oliveira e os bolsistas de iniciação científica Paola M artins Celestino, Luciana A. E. Ribeiro e Leandro Lizmeyer.
} 


\section{Lena Vania Ribeiro Pinheiro}

Este ciclo ou fluxo de comunicação e informação é mostrado por V ickery (1999), em denso e extenso artigo sobre informação científica e tecnológica, articulando história, políticas e comunicação em $C \& \mathrm{~T}$, em uma evolução histórica do século 20 até a mídia eletrônica, inter-relacionando esses diferentes, mas convergentes, aspectos.

Para Vickery (1999, p. 477), os problemas de informação científica e tecnológica são "objetivos e subjetivos", e, dentre seus questionamentos, destacamos o que denominou "contradições", nascidas da especialização na ciência e estudos multidisciplinares, o que, por sua vez, "... cria novos problemas na transferência da informação". Ele chama a atenção para a abrangência do conceito de ciência e para a existência de "cadeial corrente de informação" diferente para cada campo do conhecimento, reforçando as idéias de outros autores, no reconhecimento de padrões de comunicação específicos por área, ainda que possam existir algumas práticas comunicacionais e, conseqüentemente, informacionais comuns.

Entre fatos significativos na história da $C \& T$, são assinalados o surgimento de conselhos e centros de pesquisa, como o Centre $\mathrm{N}$ ational de la Recherche Scientifique (CN RS), em 1939, o crescimento da pesquisa industrial e 0 financiamento federal para a pesquisa, nos Estados U nidos e na G rã-Bretanha.

No ciclo da informação traçado por Vickery (1999), reproduzindo todos os seus componentes, 0 autor fez um mapeamento completo, incluindo a transferência da informação, da comunicação científica desde a comunicação informal, os meios impressos e a avaliação pelos pares, até a comunicação (e-mail, neewsgroup etc.) e informação eletrônicas (O PAC s- 0 nline Public Access C atalog) e processos como o download, que demonstram 0 entrelaçamento de recursos de comunicação e informação nas redes eletrônicas, conforme será demonstrado e discutido no presente artigo.

\section{OBJETIVOS E METODOLOGIA}

0 tema da pesquisa é muito novo, inclusive no exterior, onde estudos foram iniciados na década de 90 , enquanto no Brasil são ainda mais recentes e datam dos últimos anos, portanto, a partir de meados dos anos 90 e início do século XXI. A novidade do tema e, conseqüentemente, o seu estágio ainda incipiente, além do caráter exploratório da maioria dos estudos, em pequeno número, foram determinantes para 0 estabelecimento dos limites da pesquisa, cujo objetivo geral foi estudar 0 processo de comunicação de comunidades científicas brasileiras, em redes eletrônicas, na geração de conhecimentos, a partir do mapeamento das práticas de comunicação e informação, ou melhor, a utilização de tecnologias como correio eletrônico, listas de discussão, salas virtuais (chats) e teleconferências, bem como os recursos ou serviços de informação, entre os quais bibliotecas virtuais e digitais.

Os objetivos específicos foram identificar fatores intervenientes (facilitadores e inibidores) da comunicação e informação científicas em redes eletrônicas, sejam tecnológicos, institucionais, pessoais, de área etc., e verificar a função e importância dos diversos recursos eletrônicos na comunicação científica em rede, na sua interdependência, assim como na relação com canais de comunicação formais e informais, tradicionais ou convencionais.

Esta pesquisa é de natureza exploratória e adotou como instrumento de coleta de dados questionários, aplicados em 7.805 pesquisadores I, II e III do C N Pq, em todos os campos do conhecimento. Foram respondidos 1.426. Os resultados do levantamento, tabulação e análise de dados são referentes ao conjunto da amostra constituída por 1.307 pesquisadores, cujos questionários não apresentaram problemas no preenchimento.

\section{ESTUDOS EMPÍRIC OS NA AMÉRICA LATINA E CARIBE}

Importante pesquisa sobre comunicação e informação científicas foi realizada por Chacón \& Pingiotti na Ven ezuela, em 1993, e, para compreender os seus resultados, é necessário conhecer 0 contexto em que foi desenvolvida, o que consta de outros trabalhos, inclusive o de Pimienta \& Liendo (1993), sobre comunicação mediada por computador. Deve ser ressaltada a visão política e estratégica desses estudos, enfocando a comunicação e informação em um amplo panorama de políticas nacionais e regionais de informática, telecomunicação, comunicação e informação, o que possibilitou uma análise mais rica do processo e forneceu pertinentes e relevantes subsídios para a presente pesquisa.

No artigo de Pimienta \& Liendo (1993) sobre o estado atual e perspectivas de desenvolvimento de redes telemáticas na A mérica Latina e $C$ aribe, é descrita a Red para A mérica Latina y el Caribe, iniciativa da U nião Latina (Redalc), e a comunicação mediada por computador é apontada como "ferramenta de características notáveis". As diretrizes fundamentais da Redalc incluem aspectos organizacionais e administrativos e são as seguintes: integração regional; 


\section{Comunidades científicas e infra-estrutura tecnológica no Brasil para uso de recursos eletrônicos...}

ênfase no usuário final; conteúdo da rede; negociação com o setor de telecomunicações; backbone regional baseado em satélites (Pimienta \& Liendo, 1993). Q uanto às implicações, os autores apontam a integração regional, a supressão de distâncias, a multiplicação de intercâmbios e 0 acesso direto a fontes, além de projeções, correspondendo a grupos de trabalho distribuídos e biblioteca "eletrônica/ virtual".

Pimienta \& Liendo (1993) ressaltam, na "nova cultura", as características de compartilhamento ou solidariedade, o compromisso e a continuidade, o pluralismo e a informação transformada em ação. Os autores chegam a um diagnóstico global de comunicação mediada por computador, no qual assinalam duas épocas, sendo a primeira regida pela tecnologia e caracterizada por pouco investimento em usuários e serviços, debilidade da interface com o usuário, não-articulação de conteúdos e dificuldade de normalização. A segunda é governada pelo mercado, com "drástica mudança de enfoque", quando os "elementos importantes vão ser agora os usuários finais, os serviços, os conteúdos, as tarifas, a organização dos grupos de usuários, a participação de atores como documentalistas, pesquisadores no nível da indústria, serviços privados de informática, de comunicação e de informação". A o enfatizar as oportunidades criadas pela rede para o desenvolvimento da região, ao mesmo tempo em que enumeram os desafios para a integração regional, os autores reafirmam a sua convicção de que "a comunicação mediada por computador agrega conceitos de solidariedade e democracia ao impedir a concentração do poder associado com a informação. A natureza do sistema visto em sua globalidade promove e permite canalizar a solidariedade..." (Pimienta \& Liendo, 1993).

O trabalho de Chacón \& Pingiotti (1993) está centralizado na avaliação de impactos das redes acadêmicas e atendeu à necessidade de avaliação e de estabelecimento de diretrizes para ação. U tilizando como ambiente o Sistema A utomatizado de Información Científica y Tecnológica (Saicyt), os dois pesquisadores lançam a seguinte questão: "Q uais as mudanças radicais de comportamento dos acadêmicos a partir do momento em que se vinculam, de maneira temporal ou permanente, a uma rede?" E justificam a pergunta pelos múltiplos pontos de vista, entre os quais o educativo, 0 político e o tecnológico. Para tanto, criaram um modelo conceptual do impacto de redes, desdobrado em quatro variáveis: insumo, barreiras de comunicação, impacto primário e impacto secundário, com seus respectivos conteúdos.
Como os usuários do Saicyt estão dispersos geograficamente, a abordagem foi feita por via eletrônica, com questionários específicos para docentes, pesquisadores e especialistas do setor produtivo. Dos resultados, são extraídos os mais relevantes, na medida de sua relação com os objetivos desta pesquisa, e, no primeiro deles sobre as funções da rede, as respostas misturam atividades de comunicação, como contato com os colegas ( $91,67 \%)$, isto é, a comunicação informal entre pares, o mais alto índice, com outras atividades típicas de busca de informação, entre as quais "baixar" textos $(80,56 \%)$ e acesso a banco de dados ( $80,56 \%)$ (Pimienta \& Liendo, 1993).

Sobre a comunicação informal entre pares, voltamos a enfatizar a sua importância e predomínio, a despeito dos inúmeros canais como periódicos científicos, anais de congressos, coletân eas, livros etc.

A comunicação científica é caracterizada pela busca de maior velocidade no intercâmbio e disseminação de idéias, vantagem constatada desde a literatura clássica da área. Em estudos comparativos entre canais formais e informais, como o de M erta (1969), a qualidade dos últimos sempre foi a de maior rapidez, inclusive de feedback em relação aos meios formais.

Esta mescla de comunicação e informação demonstrada nos resultados, além de ser natural, é estimulada pelas condições da pesquisa, a partir do Saicyt, sistema que provê a conexão, simultaneamente ao acesso a serviços de informação, característica também do Prossiga Informação e Comunicação para Ciência, Tecnologia e Inovação , no Brasil.

Nos resultados referentes à área de pesquisa, é oportuno voltar a chamar a atenção para a mescla de funções e ações - associações $(90,74 \%)$, bibliografias $(80,58 \%)$, difusão de trabalhos $(58,53 \%)$, discussão $(46,3 \%)$, realização de estudos $(43,52 \%)$ até melhoramento de métodos $(26,85 \%)$ - relacionadas com a comunicação informal e informação científica que podem ser realizadas mediante contados com os pares e instituições, como as associações.

0 índice mais al to é o de associações, que representam uma das formas de institucionalização na ciência. Sociedades científicas sempre desempenharam importante papel, inclusive de veículos de disseminação da informação e do conhecimento, e isto já ocorria no século XVII, quando do surgimento das primeiras, a Royal Society of London e a Académie de France. (M eadows, 1974, 1999). 


\section{Lena Vania Ribeiro Pinheiro}

A presença de revistas eletrônicas, de debates que podem se concretizar por meio de correio eletrônico e também de listas de discussão parece indicar a adoção de recursos eletrônicos em significativa escala, o que também vai se verificar no Brasil.

Q uanto aos resultados relativos ao setor de desenvolvimento tecnológico, chamamos a atenção das funções distintas dos outros dois setores (acadêmico e de pesquisa) e suas características próprias, sobretudo vinculadas ao conhecimento, melhoramento e divulgação de produtos e mercado.

\section{Pesquisas empíricas brasileiras sobre comunicação e informação em redes eletrônicas no Brasil}

Durante 0 desenvolvimento da pesquisa, a orientação de dissertações e teses, direta ou indiretamente relacionadas à questão da pesquisa, muito contribuiu para a ampliação dos quadros teórico e empírico e o aprofundamento e enriquecimento da análise e discussão dos resultados. D entre estas, destacamos a dissertação de mestrado em ciência da informação de Job Lúcio Gomes Vieira (1998), intitulada "Correio eletrônico, 0 impacto da comunicação técnico-científica da Embrapa: um estudo de caso" e a mémoire e a monografia de Tonia $M$ acedo (1999), engenheira da Petrobrás, além das mais recentes, abordadas no último tópico deste artigo.

A dissertação de Vieira, embora o título contenha apenas correio eletrônico, na verdade abrangeu os diferentes tipos de "tecnologias de correio eletrônico", conceito que en globa o correio eletrônico pessoa a pessoa, as listas de discussão, as salas virtuais, teleconferências e boletins de aviso. $\mathrm{N}$ a pesquisa são levantados índices de uso e vantagens e desvantagens, 0 que mais interessa a este artigo. Reforçam esses resultados declarações contundentes dos pesquisadores entrevistados, sobretudo aqueles que, em regiões mais distantes dos grandes centros de pesquisa brasileiros, concentrados no Sudeste, têm na rede um recurso para minimizar as distâncias e, conseqüentemente, os desequilíbrios socioeconômicos regionais. $\mathrm{N}$ esse sentido, a pesquisa de Vieira confirma, em um país de dimensões continentais como o Brasil, a função democrática da rede e a necessidade de uma sólida infra-estrutura nacional de informação, comunicação, informática e telecomunicações.

Tratando-se de estudo realizado em uma instituição brasileira de pesquisa, os seus resultados podem traduzir uma situação nacional e chamar a atenção das instituições de nosso país para investimentos na infraestrutura de redes como decisiva ferramenta para a geração e socialização de conhecimentos e de acesso à informação científica e técnica.
A monografia de M acedo (1999) tem por título "Redes (cobrindo) o informal: a inteligên cia competitiva distribuída" e partiu dos estudos de comunicação científica e em laboratórios de $P \& D$ para a sua elaboração. A pesquisa empírica foi realizada no $C$ entro de Recursos Humanos do Sudeste CEN -SUD da Petrobras, em uma rede de especial istas em eletricidade, tendo por objetivo geral mostrar a importância das redes informais para os sistemas de inteligência competitiva. A brangeu, basicamente, informações técnicas, de equipamentos e sobre cursos, correlacionando-as aos fatores críticos de sucesso, considerando as redes eletrônicas como "espaço virtual de decisões" (Tyson, 1998). A autora conclui: “...decisões, estratégias e táticas constituem não simplesmente resultantes de processos analíticos/ técnicos, mas negociações dinâmicas e complexas tecidas pelo mesmo fio que tece a rede informal de comunicação eletrônica dos profissionais de eletricidade da Petrobras" (M acedo, 1999).

\section{REDES ELETRÔNICAS E A UTILIZAÇÃO DE RECURSOS DE COMUNICAÇÃO E INFORMAÇÃO CIENTÍFICAS}

Este capítulo de análise e discussão de resultados reúne um conjunto de dados que se inicia pelo comportamento do pesquisador diante da Internet e apresenta dois desdobramentos, ainda que sejam processos interligados: um para comunicação e outro para informação em redes eletrônicas.

A primeira pergunta trata do uso da Internet por faixa etária, cujos resultados apontam o índice maior entre 41 e 50 anos, concentrando mais da metade das respostas. Isto é interessante observar, por ser a Internet recente, e, portanto, pesquisadores nessa faixa não cresceram em uma cultura de rede e, muitas vezes, não têm familiaridade com essa tecnologia. A faixa etária entre 51 e 60 anos é expressiva e ocupa o segundo lugar no uso, e a presença de pesquisadores de 20 a 30 anos é pouco significativa, o que demonstra a pequena participação de jovens cientistas na comunidade brasileira.

Este resultado extrapola os objetivos da pergunta e traduz a situação de órgãos públicos federais brasileiros, nos quais os concursos ficaram suspensos por muitos anos e a reabertura recente foi insuficiente em relação às necessidades de renovação de quadros, situação na qual se inserem o M inistério de Ciência e Tecnologia (M CT) e seus institutos de pesquisa, o CN Pq e o M inistério da Educação e professores universitários, instituições em que exercem atividades os pesquisadores abordados nesta pesquisa. 


\section{Comunidades científicas e infra-estrutura tecnológica no Brasil para uso de recursos eletrônicos...}

Perguntou-se aos pesquisadores sobre o início do uso de rede eletrônica, e não especificamente a Internet, pois antes existiu a Bitnet; daí devermos mencionar a primeira dissertação relativa a essa tecnologia, sobre comunicação científica em rede eletrônica, apresentada por Figueira N etto (1994) no Programa de Pós-G raduação em Ciência da Informação do Ibict, em convênio com a U FRJ.

$\mathrm{N}$ a figura 1, os resultados são coerentes com a trajetória das redes eletrônicas no Brasil, as primeiras surgidas na década de 80, e o início da implantação da Rede $\mathrm{N}$ acional de Pesquisas (RNP) no começo dos anos 90, mas lançada em 1989 (Takahashi, 1992). Portanto, é natural a convergência de uso na década de 90 , quando - Brasil ingressa de fato na Internet, época em que também nasceu o Prossiga, que passou a oferecer serviços eletrônicos de informação e comunicação para as comunidades científicas brasileiras e, de certa forma, preencheu uma lacuna nesse tipo de atuação, no Brasil, por parte de órgãos públicos.

A próxima questão está estreitamente relacionada à primeira e indica a freqüência e tempo de uso, ou melhor, se é uma prática já incorporada às atividades de pesquisadores. Os resultados confirmam essa hipótese, em um percentual de regularidade e, especificamente, de uso diário muito alto por quase a totalidade (96\%), de acordo com a figura 2 .

A disponibilidade da tecnologia é importante para se criar 0 hábito, e alguns autores, entre os quais Abels, Liebscher \& Denman (1996), destacam o acesso a estes meios como fator determinante para 0 uso da rede, 0 que é uma conclusão natural; enfatizam, porém, a importância da proximidade física e o uso nãocompartilhado, em estações de trabalho. Estas facilidades dependem da infra-estrutura tecnológica existente na instituição, para assegurar que cada pesquisador possa usar individualmente o "seu" computador, disponível em sua sala de trabalho.

As atividades de pesquisa, no Brasil, no caso do órgão nacional de fomento, o CN Pq, podem ser efetivadas, na sua maioria, em procedimentos via rede eletrônica: 0 Curriculum Lattes, adotado para avaliação do pesquisador, o Diretório de G rupos de Pesquisa, no qual estão registrados os pesquisadores brasileiros, distribuídos pelas suas respectivas linhas de pesquisa, 0 preenchimento de formulários e encaminhamento de projetos de pesquisa. Já existe, portanto, infra-estrutura tecnológica que permite, inclusive, o acompanhamento de resultados, acesso a informações sobre alocação de recursos e distribuição de bolsas, enfim, há transparência no processo de fomento (Pinheiro, 2000).
FIGURA 1

\section{Década de início do uso de rede eletrônica}

Não respondido

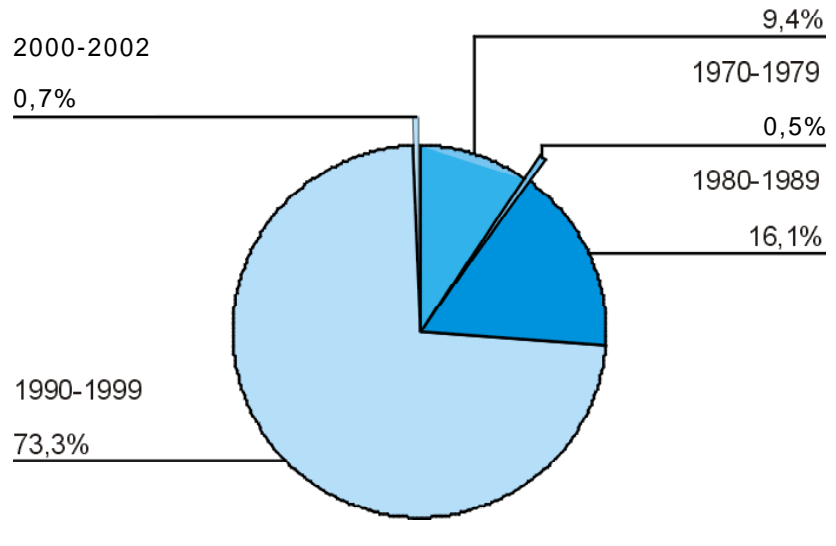

FIGURA 2

\section{Freqüência de uso da Internet}

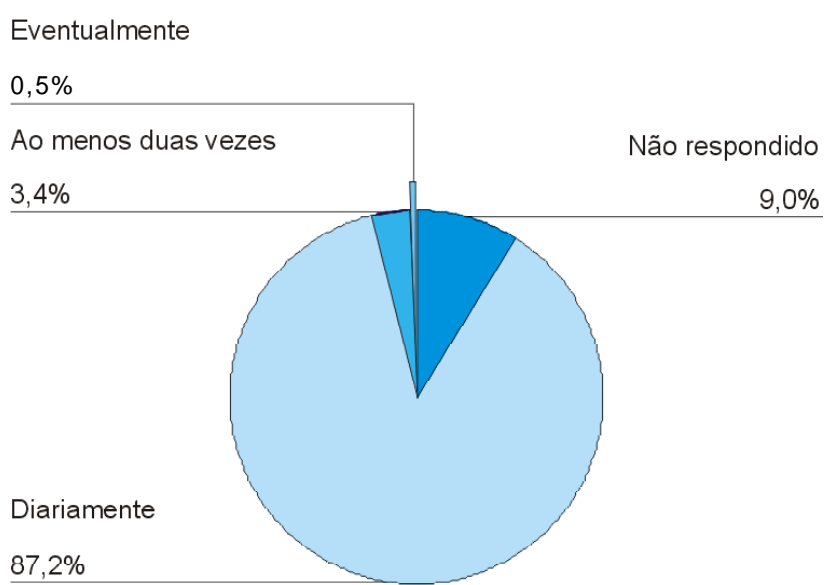

Quanto ao uso da rede, se no local de trabalho ou nas residências, as respostas dos pesquisadores demonstram o uso simultâneo, tanto na instituição quanto na sua residência, com índice mais alto, $62,4 \%$, seguido pela utilização apenas na instituição, praticamente a metade $(31,8 \%)$.

Este resultado chama a atenção, novamente, para a importância de investimentos na infraestrutura de rede, por parte das instituições de ensino e pesquisa, para Internet e intranet. O utro aspecto relevante é que os respondentes, por estarem fortemente engajados em pesquisa, precisam, conseqüentemente, de informações para se manterem atualizados, conhecer os avanços do seu campo e os pesquisadores nele atuantes. 
TABELA 1

Fatores intervenientes na comunicação e transferência da informação

\begin{tabular}{|c|c|c|c|c|c|c|c|c|c|c|c|c|}
\hline & \multicolumn{2}{|c|}{$\begin{array}{l}\text { Problemas de } \\
\text { conexão }\end{array}$} & \multicolumn{2}{|c|}{$\begin{array}{c}\text { Falta de suporte } \\
\text { técnico }\end{array}$} & \multicolumn{2}{|c|}{$\begin{array}{c}\text { Não- } \\
\text {-familiaridade com } \\
\text { o soflumare }\end{array}$} & \multicolumn{2}{|c|}{$\begin{array}{l}\text { Barreira } \\
\text { lingüística }\end{array}$} & \multicolumn{2}{|c|}{$\begin{array}{l}\text { Falta de } \\
\text { treinamento }\end{array}$} & \multicolumn{2}{|c|}{ Outros fatores } \\
\hline & $\mathrm{N}$ & $\%$ & $\mathrm{~N}$ & $\%$ & $\mathrm{~N}$ & $\%$ & $\mathrm{~N}$ & $\%$ & $\mathrm{~N}$ & $\%$ & $\mathrm{~N}$ & $\%$ \\
\hline $\begin{array}{l}\text { Muito } \\
\text { relevante }\end{array}$ & 383 & $49,1 \%$ & 153 & $21,3 \%$ & 75 & $10,8 \%$ & 27 & $4,2 \%$ & 67 & $10,2 \%$ & 39 & $28,9 \%$ \\
\hline Relevante & 1.37 & $17,6 \%$ & 199 & $27,7 \%$ & 126 & $18,2 \%$ & 65 & $10,1 \%$ & 110 & $16,7 \%$ & 19 & $14,1 \%$ \\
\hline $\begin{array}{l}\text { Pouco } \\
\text { rclevantc }\end{array}$ & 131 & $16,8 \%$ & 180 & $25,0 \%$ & 220 & $31,8 \%$ & 119 & $18,4 \%$ & 157 & $23,8 \%$ & 19) & $14,1 \%$ \\
\hline Irrelevante & 129 & $16,5 \%$ & 187 & $26,0 \%$ & 271 & $39,2 \%$ & 434 & $67,3 \%$ & 325 & $49,3 \%$ & 58 & $43,0 \%$ \\
\hline Total & 780 & $100,0 \%$ & 719 & $100,0 \%$ & 692 & $100,0 \%$ & 645 & $100,0 \%$ & 659 & $100,0 \%$ & 135 & $100,0 \%$ \\
\hline
\end{tabular}

$M$ as não basta ter disponível microcomputador e estrutura de rede eletrônica e acesso à Internet, pois o bom uso da rede depende também de certos conhecimentos, habilidades e outros fatores, identificados na tabela 1.

Esses problemas tanto podem ser eminentemente de infraestrutura tecnológica, entre os quais os de con exão, e até de telefonia, quanto de habilidades para lidar com as tecnologias, e sobre essa questão existem muitos trabalhos publicados na literatura da área. Há, também, uma dificuldade que já existia em relação a publicações impressas: 0 desconhecimento de línguas estrangeiras, principalmente 0 inglês, que predomina nas ciências, em geral, e também na Internet.

Pelos resultados mostrados, problemas de conexão aparecem como os mais fortes, questão decorrente, sobretudo, de falta de política nacional para o setor, secundada pela política institucional visando a uma sólida e potente infra-estrutura de comunicação eletrônica, acompanhando a evolução tecnológica e ingressando na Internet 2 . A segunda dificuldade, de falta de suporte técnico, é mais institucional e depende da capacidade dos laboratórios e setores de computação eletrônica e rede e do pessoal neles alocados para apoiar atividades científicas, inclusive promover treinamentos e manter assistência regular.

Tratando-se de uma comunidade de pesquisadores, era de se esperar problemas em menor nível com a barreira lingüística, ainda presente, mas não relevante para a maioria. Dependendo de cada área do conhecimento e da concentração maior ou menor da produção científica em determinado país, as exigências também variam. $\mathrm{Na}$ ciência da informação, por exemplo, a produção científica está concentrada na língua inglesa, seja proveniente dos Estados U nidos ou da Grã-Bretanha. Portanto, não possuir domínio da língua inglesa, pelo menos para leitura, levará também ao desconhecimento de parte significativa dos avanços da área.

Em termos de feedback para instituições brasileiras, foi importante identificar os problemas existentes no uso dos recursos eletrônicos, uma vez que alguns podem ser minorados ou solucionados pelo apoio técnico da instituição do pesquisador e até de políticas públicas nacionais, pois foi mencionado, além dos problemas listados no questionário, o alto custo da telefonia.

Alguns autores defendem que com a rede não houve apenas a ampliação de canais e a criação de novos, 0 que aperfeiçoa as práticas dos "colégios invisíveis" ou ciberspace colleges, mas, conforme acredita $\mathrm{G}$ resham Jr. (1998), ocorreu algo radicalmente novo, pela conf luência e coexistência de texto, velocidade e interatividade. Por outro lado, a comunicação eletrônica recupera, de certa forma, a interatividade da comunicação face a face (Levinson apud Gresham Jr., 1998).

Sobre a expansão do grau de comunicação entre pesquisadores, os impactos da rede são insofismáveis, e diversas pesquisas, entre as quais as brasileiras, confirmam esta vantagem, a superação das distâncias geográficas, o que é um fator considerável em países de dimensões continentais como o Brasil.

A pesquisa de Vieira (1998), por exemplo, comprova a repercussão da rede na integração institucional e nas relações interinstitucionais e no encurtamento das distâncias, fatores importantes para uma in stituição como a Embrapa, ambiente de seu estudo, e muitas instituições nacionais com representações dispersas geograficamente, em todas as regiões e estados brasileiros. 
Comunidades científicas e infra-estrutura tecnológica no Brasil para uso de recursos eletrônicos...

TABELA 2

Finalidades de uso da Internet em atividades científicas

\begin{tabular}{|l|r|r|r|r|c|c|}
\hline \multicolumn{1}{|c|}{ Finalidades de uso } & \multicolumn{2}{c|}{ Respondidos } & \multicolumn{2}{c|}{$\begin{array}{c}\text { Não } \\
\text { respondidos }\end{array}$} & \multicolumn{3}{c|}{ Total } \\
\hline & \multicolumn{1}{|c|}{$\mathrm{N}$} & $\%$ & $\mathrm{~N}$ & $\%$ & $\mathrm{~N}$ & $\%$ \\
\hline Comunicação entre pares & 1260 & $96,4 \%$ & 47 & $3,6 \%$ & 1307 & $100,0 \%$ \\
\hline Comunicação com pesquisadores de outras áreas & 960 & $73,5 \%$ & 347 & $26,5 \%$ & 1307 & $100,0 \%$ \\
\hline Comunicação para fins didáticos & 1164 & $89,1 \%$ & 143 & $10,9 \%$ & 1307 & $100,0 \%$ \\
\hline Circulação de trabalhos científicos antes da publicação & 986 & $75,4 \%$ & 321 & $24,6 \%$ & 1307 & $100,0 \%$ \\
\hline Submissão de trabalhos a pcriódicos & 1090 & $83,4 \%$ & 217 & $16,6 \%$ & 1307 & $100,0 \%$ \\
\hline Submissão de trabalhos a congressos & 1209 & $92,5 \%$ & 98 & $7,5 \%$ & 1307 & $100,0 \%$ \\
\hline Outras finalidades & 819 & $62,7 \%$ & 488 & $37,3 \%$ & 1307 & $100,0 \%$ \\
\hline
\end{tabular}

Ainda sobre essa questão, Archambault (1995) destaca que a dispersão geográfica favorece a utilização da rede. No caso de comunicação intra-institucional, devemos chamar a atenção para a situação mencionada, de instituições de âmbito nacional, porque, assim, pesquisadores de regiões periféricas aos grandes centros são muito beneficiados com esse recurso.

Sobre a importância das redes eletrônicas em países em desenvolvimento, Pimienta \& Liendo (1993) consideram que, em termos regionais, em face dos desequilíbrios socioeconômicos, esta tecnologia pode contribuir para minimizar a concentração de poder, na medida em que democratiza 0 acesso à informação.

A pergunta seguinte, visualizada na tabela 2 , diz respeito aos objetivos dos pesquisadores ao usarem a Internet para fins de atividades científicas.

O s maiores índices estão relacionados à própria comunicação científica entre pares $(96,4 \%)$, seguida do encaminhamento de trabalhos a congressos $(96,5 \%)$, alcançando alto índice também a submissão de artigos para periódicos $(83,4 \%)$. É oportuno chamar a atenção que a rede eletrônica tanto estimula a comunicação informal, entre pares, quanto formal, facilitando os procedimentos de avaliação em periódicos e congressos, com resultados semelhantes aos de Chacón $\&$ Pingiotti (1993).

A parece, com percentual de 75,4\%, a circulação de trabalhos científicos antes de sua publicação, as prépublicações (pre-print), prática comum entre pesquisadores que integram "colégios invisíveis", uma das formas mais freqüentes de comunicação informal (M erta, 1972; Price, 1976). Portanto, esse procedimento se manteve ao longo dos anos, apenas atualmente mediado por uma nova tecnologia.
0 utro resultado significativo diz respeito às atividades docentes, em um percentual de $89,1 \%$, o que, tratandose de pesquisadores, é natural pela dupla atividade de ensino e pesquisa, principalmente na pós-graduação ou, ainda, em cursos a distância ou semipresenciais, o que, não sendo objetivo da presente pesquisa, não foi verificado.

As perguntas referentes à tabela 2 foram, propositadamente, voltadas à comunicação, não incluindo informação, que faz parte de outro quadro, ainda que venha sendo ressaltada, no decorrer deste artigo, a confluência da comunicação e informação. $\mathrm{N}$ a pergunta aberta sobre outras finalidades de uso da Internet, muito natural mente aparecem, também, serviços e produtos de informação, assim apontados pelos pesquisadores: revisão bibliográfica, obtenção de dados secundários para investigação, pesquisa bibliográfica, pesquisa no M edline, organização de eventos científicos, acesso e cópia de artigos científicos completos disponibilizados na rede, acesso a instrumentos de pesquisa como questionários, acesso a sites de fontes financiadoras de pesquisa, lista de debates, envio e recebimento de arquivos entre a casa e 0 trabalho, troca de dados com parceiros remotos, elaboração de artigos em parceria, pesquisa de algumas fontes de dados, comunicação formal com parceiros de projetos, dentre outros.

O s resultados, mais uma vez, são altamente afirmativos, consolidam os anteriores e podem ser exemplificados com 0 relato de experiência de um dos pesquisadores abordados: "Já publiquei dois artigos, totalmente discutidos através da Internet com um inglês e uma italiana quenunca vi pessoalmente. V amos publicar outro esse ano com uma argentina". 
TABELA 3

U so de tecnologias de comunicação eletrônica

\begin{tabular}{|c|c|c|c|c|c|c|c|c|c|c|c|c|c|c|}
\hline & \multicolumn{2}{|c|}{$\begin{array}{l}\text { Correio } \\
\text { Hiletrônico }\end{array}$} & \multicolumn{2}{|c|}{$\begin{array}{l}\text { Lista de } \\
\text { discussão }\end{array}$} & \multicolumn{2}{|c|}{ Teleconferência } & \multicolumn{2}{|c|}{$\begin{array}{l}\text { Salas Virtuais } \\
\text { (Chats) }\end{array}$} & \multicolumn{2}{|c|}{ Newsgroup } & \multicolumn{2}{|r|}{ IRC } & \multicolumn{2}{|c|}{$\begin{array}{c}\text { Outras } \\
\text { tecnologias }\end{array}$} \\
\hline & $\mathrm{N}$ & $\%$ & $N$ & $\%$ & $\mathrm{~N}$ & $\%$ & $\mathrm{~N}$ & $\%$ & $\mathrm{~N}$ & $\%$ & $\lambda^{-}$ & $\%$ & $\mathrm{~N}$ & $\%$ \\
\hline $\begin{array}{l}\text { Muito } \\
\text { relevante }\end{array}$ & 1082 & $94,9 \%$ & 88 & $9,4 \%$ & 33 & $4,2 \%$ & 18 & $2,2 \%$ & 21 & $2,7 \%$ & 15 & $2,6 \%$ & 61 & $24,9 \%$ \\
\hline Relevante & 37 & $3,2 \%$ & 311 & $33,0 \%$ & 128 & $16,1 \%$ & 44 & $5,4 \%$ & 140 & $18,0 \%$ & 39 & $6,7 \%$ & 69 & $28,2 \%$ \\
\hline $\begin{array}{l}\text { Pouco } \\
\text { relevante }\end{array}$ & 2 &, $2 \%$ & 267 & $28,4 \%$ & 188 & $23,7 \%$ & 132 & $16,3 \%$ & 215 & $27,7 \%$ & 99 & $16,9 \%$ & 31 & $12,7 \%$ \\
\hline Irrelevante & 19 & $1,7 \%$ & 275 & $29,2 \%$ & 444 & $56,0 \%$ & 614 & $76,0 \%$ & 400 & $51,5 \%$ & 433 & $73,9 \%$ & 84 & $34,3 \%$ \\
\hline Total & 1140 & $100,0 \%$ & 941 & $100,0 \%$ & 793 & $100,0 \%$ & 808 & $100,0 \%$ & 776 & $100,0 \%$ & 586 & $100,0 \%$ & 24.5 & $100,0 \%$ \\
\hline
\end{tabular}

TABELA 4

Tipo de participação em listas discussão e salas virtuais

\begin{tabular}{|l|c|c|c|c|c|c|}
\hline & \multicolumn{2}{|c|}{ Respondidos } & \multicolumn{2}{|c|}{ Não respondidos } & \multicolumn{2}{|c|}{ Total } \\
\hline Tipo de participação & $\mathrm{N}$ & $\%$ & $\mathrm{~N}$ & $\%$ & $\mathrm{~N}$ & $\%$ \\
\hline Recebe e transmite informação & 281 & $21,5 \%$ & 1026 & $78,5 \%$ & 1307 & $100,0 \%$ \\
\hline Só recebe informações & 116 & $8,9 \%$ & 1191 & $91,1 \%$ & 1307 & $100,0 \%$ \\
\hline Participa das discussõcs & 187 & $14,3 \%$ & 1120 & $85,7 \%$ & 1307 & $100,0 \%$ \\
\hline$\Lambda$ penas acompanha as discussões & 211 & $16,1 \%$ & 1096 & $83,9 \%$ & 1307 & $100,0 \%$ \\
\hline Outras formas de participação & 57 & $4,4 \%$ & 1250 & $95,0 \%$ & 1307 & $100,0 \%$ \\
\hline
\end{tabular}

Ao lado da expansão dos grupos de pesquisa, a Internet cria ou estimula a ampliação das relações interdisciplinares entre campos do conhecimento, e tem sido comprovado o papel da rede nesse processo, não somente conectando pessoas, mas também disciplinas, nas suas diferentes interfaces, além de múltiplas abordagens. Hert (1997) ressalta a possibilidade de mútuas influências entre pesquisadores, com também múltiplas perspectivas.

Os diversos meios de comunicação eletrônica utilizados por pesquisadores brasileiros podem ser vistos na tabela 3, com a indicação de sua relevância.

Os resultados reforçam os de pesquisas da área, inclusive as brasileiras, nas quais o meio de comunicação eletrônica mais utilizado é o correio eletrônico, com larga diferença dos demais, secundado pelas listas de discussão, conforme ocorreu no estudo de Vieira (1998), na Embrapa. As demais tecnologias (listas de discussão, teleconferências, salas virtuais, newgroups) apresentam índices mais baixos, o que demonstra a necessidade, no Brasil, de sua ampla difusão, e entre "outras tecnologias" foram indicadas bibliotecas virtuais, ICQ e FTP.
O correio eletrônico, que inicialmente parecia ser um substituto dos meios de comunicação tradicional, entre os quais cartas, memorandos e telefonemas, adquiriu características próprias e assumiu um papel ímpar na comunicação (Burton, 1994). Entre as vantagens apontadas por diversos autores, está o fato de ser um "meio basicamente textual" (M arkus, 1992, apud Burton) e utilizar linguagem informal, semelhante à discussão acadêmica verbal (Yates \& O rlikovski, 1992 apud Burton), além da assincronicidade (Burton, 1994).

0 índice de utilização de listas de discussão, conforme visto, foi o segundo mais importante (com indicação de muito relevante e relevante), alcançando um percentual de $42,5 \%$, o que ensejou uma pergunta, juntamente com salas virtuais, embora estas tenham sido consideradas pouco relevantes ou irrelevantes em alto índice, 92,4\%, e os resultados sobre o tipo de participação nesses meios de comunicação eletrônica são apresentados na tabela 4.

O s resultados apontam a participação ativa (recebendo e transmitindo) em listas de discussão e salas virtuais, pelos poucos $(21,5 \%$ ) pesquisadores respondentes, o que pode estar relacionado à experiência em lidar com esta tecnologia, principalmente a sala virtual, que, por ser sincrônica, exige habilidade e respostas imediatas. $\mathrm{Na}$ pergunta seguinte, o período de tempo adotado para cada 


\section{Comunidades científicas e infra-estrutura tecnológica no Brasil para uso de recursos eletrônicos...}

resposta foi o seguinte: usuário novo (até seis meses), usuário intermediário (até um ano), usuário antigo (mais de um ano), (figura 3).

0 índice de ausência de resposta a esta pergunta, muito alto, praticamente a metade $(50,3 \%)$ é, por si só, in dicador da ainda pequena utilização desses meios eletrônicos, principalmente as salas virtuais, ou até do seu desconhecimento por parte dos pesquisadores ou de sua não-disponibilidade, ratificando observações anteriores.

Considerando-se que a comunicação informal predomina na rede, principalmente por meio do correio eletrônico, este tópico é encerrado com algumas considerações de autores da literatura da área e também de pesquisadores abordados nesta pesquisa.

Sobre possíveis alterações na comunicação informal, Levinson (1990, apud G resham Jr, 1994) faz interessantes observações comparando os meios formais impressos aos canais informais, e cita as conferências eletrônicas como uma evolução na interatividade da comunicação face a face, embora também apresentem características de permanência, própria da comunicação textual.

Ao justificarem as suas respostas sobre comunicação informal, os pesquisadores apontaram diversas vantagens, entre as quais, rapidez e recuperação da linguagem escrita na comunicação pessoal e a já tão enfatizada facilidade na comunicação, conforme a seguinte afirmativa:

"Hoje posso enviar documentos para meus amigos analisarem e opinarem de forma segura e rápida, sem contar que ficou muito mais barato, tanto com relação ao telefone quanto aos correios".

Inversamente, outros respondentes apontaram problemas como o pequeno número de pesquisadores que têm acesso à Internet, enquanto um chamou a atenção para a importância dos meios mais tradicionais, expressa pela seguinte frase: "Ainda mandamos carta pelo correio, ainda participamos de reuniões científicas". Portanto, aparece não somente a comparação com os meios tradicionais, mas também com a comunicação informal interpessoal, conforme pode ser observado nas falas de pesquisadores:

"U tilizo a Internet como ferramenta para agilizar contatos científicos e intercâmbio profissional, mas não como substituto de contatos interpessoais", ou, na mesma linha de raciocínio, "a comunicação informal continua sendo importante".

Os resultados apresentados a seguir foram direcionados às atividades de informação científica e tecnológica e à
FIGURA 3

Tempo de participação em lista de discussão e sala virtual

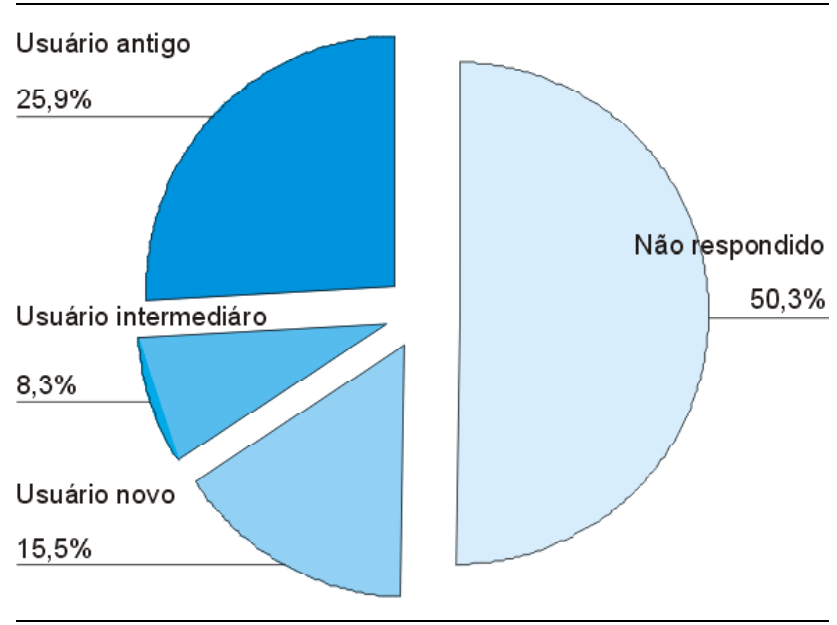

FIGURA 4

Recurso inicial de busca à informação

Biblioteca

$22,7 \%$

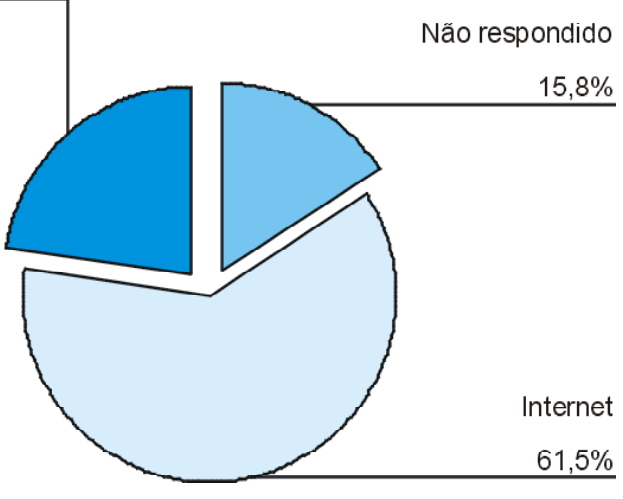

comparação de uso entre documentos impressos e eletrônicos (figura 4).

A primeira pergunta pretendia medir não somente a importância da Internet para as atividades de pesquisa, mas a sua prioridade ou não em relação aos recursos impressos.

As respostas apontam a prioridade da Internet, o que pode ser comparado com resultados de estudos de usuários de bibliotecas tradicionais, nos quais a utilização depen dia da proximidade física da biblioteca, isto é, eram primeiramente consultadas as local izadas mais perto. Esta condição pode determinar o uso prioritário da Internet, o que não significa menor importância de acervos impressos. 
TABELA 5

Relevância de serviços e produtos de informação eletrônicos

\begin{tabular}{|c|c|c|c|c|c|c|c|c|c|c|c|c|}
\hline & \multicolumn{2}{|c|}{ Base de Dados } & \multicolumn{2}{|c|}{ Bibliografias } & \multicolumn{2}{|c|}{ Sites de eventos } & \multicolumn{2}{|c|}{$\begin{array}{c}\text { Bibliotecas digitais } \\
\text { c virtuais }\end{array}$} & \multicolumn{2}{|c|}{$\begin{array}{l}\text { Aquisição de } \\
\text { publicações }\end{array}$} & \multicolumn{2}{|c|}{$\begin{array}{l}\text { Outros serviços e } \\
\text { produtos de } \\
\text { informação }\end{array}$} \\
\hline & $-\mathrm{T}$ & $\%$ & $\mathrm{~N}$ & $\%$ & $\mathrm{~N}$ & $\%$ & $\mathrm{~N}$ & $\%$ & $\mathrm{~N}$ & $\%$ & $\mathrm{~N}$ & $\%$ \\
\hline Muito relcvantc & 591 & $61,2 \%$ & 661 & $67,4 \%$ & \begin{tabular}{|l|}
447 \\
\end{tabular} & $45,2 \%$ & 498 & $51,0 \%$ & 344 & $36,7 \%$ & 5) & $31,2 \%$ \\
\hline Relevanle & 238 & $24,7 \%$ & 212 & $21,6 \%$ & 380 & $38,4 \%$ & 302 & $30,9 \%$ & 300 & $32,0 \%$ & 50 & $26,5 \%$ \\
\hline \begin{tabular}{|l|} 
Pouco \\
relevante
\end{tabular} & 90 & $9,3 \%$ & 69 & $7,0 \%$ & 120 & $12,1 \%$ & 129 & $13,2 \%$ & 200 & $21,3 \%$ & 42 & $22,2 \%$ \\
\hline Irrelevante & 46 & $4,8 \%$ & 38 & $3,9 \%$ & 42 & $4,2 \%$ & 48 & $4,9 \%$ & 93 & $9,9 \%$ & 38 & $20,1 \%$ \\
\hline Total & 965 & $100,0 \%$ & 980 & $100,0 \%$ & 989 & $100,0 \%$ & 977 & $100,0 \%$ & 937 & $100,0 \%$ & 189 & $100,0 \%$ \\
\hline
\end{tabular}

A comparação entre o uso de fontes impressas e eletrônicas por pesquisadores brasileiros é apresentada na figura 5.

Os resultados indicam a predominância do uso dos recursos eletrônicos, com uma diferença acentuada para os tradicionais, em papel e, menor ainda, o uso simultâneo do eletrônico e do impresso.

Q uanto aos serviços eletrônicos de informação utilizados, a tabela 5 permite a sua identificação e também a sua importância para atividades de pesquisa.

Os resultados mostram a utilização dos que já existiam antes da Internet e passaram a ficar disponíveis na rede, como as bases de dados e bibliografias, além de informações sobre eventos científicos, juntamente com produtos típicos da rede, como bibliotecas virtuais. Convém alertar sobre os conteúdos ou abrangência de bibliotecas virtuais e digitais, que podem incluir bases de dados, inclusive sobre eventos, bibliografias etc.

Por outro lado, a nomenclatura de serviços e produtos de informação na Internet é ainda muito nova e, às vezes, desconhecida do pesquisador. Há, por exemplo, mesmo na área de ciência da informação, em que esses conceitos são estudados, diferentes interpretações, sobretudo de bibliotecas virtuais e bibliotecas digitais, daí não ter sido feita distinção, nesta pesquisa, e ambas foram reunidas no questionário.

Conforme podemos observar, os serviços e produtos de informação praticamente se equivalem em importância, uma vez que os percentuais de muito relevante e relevante são os seguintes: bibliografias: $89,1 \%$; bases de dados: $85,9 \%$; sites de eventos: $83,7 \%$; bibliotecas virtuais: $81,9 \%$.

É oportuno esclarecer a proximidade conceitual entre bibliografias, bases de dados e bibliotecas virtuais/ digitais, mais acentuada na forma eletrônica, quando esses recursos podem assumir um certo hibridismo ou fusão.
FIGU RA 5

U tilização de recursos impressos e eletrônicos em pesquisas

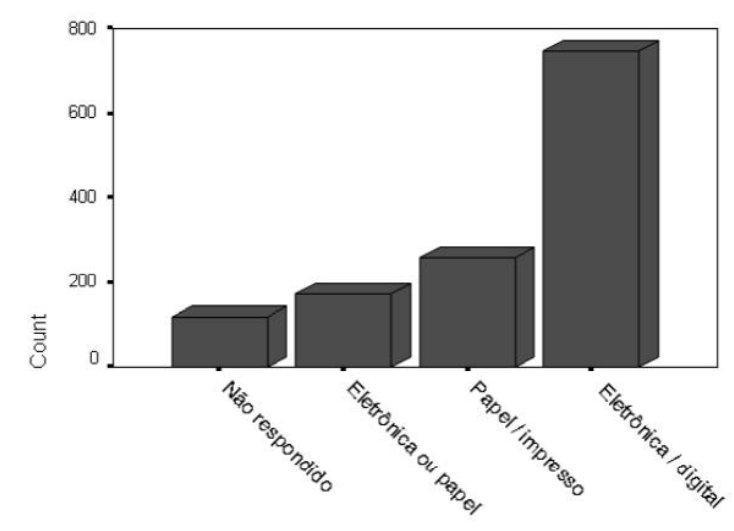

A base de dados, por exemplo, pode corresponder a uma bibliografia, bem como a biblioteca virtual ou digital pode incluir bibliografias ou bases de dados. Portanto, as fronteiras conceituais tornam-se mais tênues na rede, o que, para o pesquisador leigo nas questões de informação, pode ter dificultado a resposta, obscurecendo a importância desses recursos para a pesquisa.

\section{PERCEPÇ ÕES E PERSPECTIVAS}

As percepções e perspectivas estão relacionadas tanto aos estudos teóricos e empíricos realizados durante 0 desenvolvimento desta pesquisa quanto aos seus resultados. U m dos primeiros fenômenos observados, decorrente da Internet, foi a aproximação entre comunicação científica (de cientistas para cientistas) e divulgação científica, termo adotado no Brasil, cuja denominação na França é vulgarização da ciência e, em outros países, principalmente da América Latina, popularização da ciência (comunicação de cientistas para a sociedade em geral). 


\section{Comunidades científicas e infra-estrutura tecnológica no Brasil para uso de recursos eletrônicos...}

U m bom exemplo desta convergência é dado na área de geologia, em relação a um evento, no caso, um terremoto ocorrido em Gujarat, na Índia, relatado em artigo de Schweig e colaboradores (2001) enfocando a agitação causada nesse evento internacional pela Internet, bem como em jornais populares, rádio e televisão. 0 foco central é uma lista regional de mala direta (mailing list), na coordenação da resposta científica, na comunicação de resultados e no intercâmbio de idéias.

Os autores observam que a necessidade de rápida e eficiente comunicação quando da ocorrência de terremotos é óbvia, bem como a oportunidade, sem precedentes, de intercâmbio de informações, e acrescentam que "... se 0 dado inclui observações de campo potencialmente efêmeras, a rápida mobilização pode ser crítica". Além disso, afirmam em seu artigo que muitos cientistas souberam desse terremoto primeiramente pela imprensa popular ou por listas que notificam fatos sobre terremotos aos seus participantes. O utra característica apontada é que estas listas podem adquirir novas funções, como, por exemplo, de clearinghouse de informações retrospectivas sobre terremotos ou de canal de informação, no qual os cientistas enviam as referências preferidas de publicações científicas, fontes e sites (Schweig et alii, 2001, p.7)

Há vantagens e desvantagens, e são apontadas, entre as primeiras, o custo mínimo da Central U nited States Earthquarke $\mathrm{H}$ azard $\mathrm{M}$ ailing List e, entre as desvantagens, 0 fato de a Internet permitir a disseminação de notícias não qualificadas ou distorcidas, daí a necessidade de distinguir fontes confiáveis ou não (Schweig et alii 2001, p.2).

Esses estudos têm evoluído bastante e, recentemente, sobretudo pela utilização de recursos de rede eletrônica, vêm despertando maior interesse, inclusive no Brasil (Pereira \& Pinheiro, 2000). Comprovam esta afirmativa cursos em diferentes universidades, no exterior, de graduação e pós-graduação, assim como sociedades científicas, institutos e projetos em comunicação científica e técnica, principalmente nos Estados U nidos e Austrália. Ao mesmo tempo, podemos observar que o despertar para o estudo da comunicação científica e tecnológica, em função da Internet, pode restringir o escopo dessas pesquisas, pelo desconhecimento da fundamentação histórica e teórica dada pela história e sociologia da ciência. É emblemático 0 trabalho de M eadows (1974), autor clássico da área, mas que vem acompanhando a sua evolução e influência das tecnologias de rede e cujo livro traz atualizações, inclusive a edição brasileira (M eadows,1999), além de artigos sob esse enfoque (M eadows e Buckle,1992).
No Brasil, no Ibict, a comunicação científica integra 0 núcleo de disciplinas de seu Programa de Pós-G raduação em Ciência da Informação, introduzida pela professora H agar Espanha Gomes na década de 70, e essa tradição pode ser comprovada pelas inúmeras dissertações sobre este tema. Tal como no exterior, nos anos 90 o escopo dessas pesquisas tem se estendido até as redes eletrônicas, conforme é evidenciado nas dissertações e teses mais recentes, algumas já mencionadas, além da tese de Sandra Rebel Gomes (2002), sobre os recursos de informação e comunicação de bibliotecas virtuais para pesquisa científica, e as dissertações de M iguel Angel Schindt (2002) e M ichell 0. Xavier da Costa (2003), ambas tratando de bibliotecas virtuais para comunidades científicas, a primeira em geociências e, a segunda, em manejo florestal sustentável.

$O$ utra conseqüência da Internet e $W$ eb ressaltada em diferentes momentos deste artigo diz respeito à confluência da comunicação e informação, processos praticamente indissociados, mas que, com as tecnologias de rede, passaram a assumir as mesmas funções, independentemente de terem sido criados para um determinado objetivo, na sua origem. U m bom exemplo são as listas de discussão, que têm por finalidade, como o próprio nome diz, o debate de questões científicas, mas que, ao mesmo tempo, são um rico meio de circulação de informação sobre publicações, eventos e recursos de informação em geral.

Especificamente sobre os resultados desta pesquisa, a comunidade de pesquisadores brasileiros parece ter incorporado, no seu cotidiano científico, as tecnologias de rede, na ação de desenvolver pesquisas e gerar conhecimentos, e tem consciência dos impactos decorrentes das redes eletrônicas, favorecendo a expansão das comunidades cientificas, facilitan do e intensificando a comunicação e ampliando 0 acesso aos diversos recursos de informação criados na rede.

A comunicação informal mantém sua predominância e é expandida, agora por meio de computadores e via rede, tendo o correio eletrônico como recurso principal, secundado pelas listas de discussão. Entre os recursos construídos de forma digital ou virtual, são relevantes os sites de eventos eas bibliotecas virtuais e digitais, muito utilizados por pesquisadores brasileiros.

O s resultados desta pesquisa podem sensibilizar as instituições de ensino, pesquisa e desenvolvimento do país para maiores investimentos na infra-estrutura tecnológica indispensável na utilização de recursos de comunicação e informação el etrônicos pela comunidade 


\section{Lena Vania Ribeiro Pinheiro}

científica brasileira e, ao mesmo tempo, para debater e demonstrar a importância dessa questão, a fim de incorporá-la às políticas públicas brasileiras de ciência e tecnologia.

\section{REFERÊN CIAS}

ABELS, Eileen G.; LIEBSCHER, Peter; DENMAN, Daniel W. Factors that influence the use of electronic networks by science and engineering faculty at small institutions. Part 1. Q ueries. Journal of the American Society for Information Science, N ew York, v. 47, n. 2, p. 146-158, 1996

ARCHAMBAULT, Caroline. La communication dans un groupe de discussion scientifique: analyse du groupe de discussion Biomch-L. Cursus, v. 1, n. 2, Printemps, 1995.

BURTON, Paul F. Electronic mail as an academic discussion forum. Journal of Documentation. London, v. 50, n. 2, p. 99-110, June 1994.

CHACÓN, Fábio; PINGIOTTI, Beatriz. Evaluando el impacto de las redes académicas: un estudio de caso. In: U na nueva manera de comunicar el conocimiento. Caracas : C resalc, 1993. p. 119-136.

COSTA, Michell Olívio Xavier da. Biblioteca virtual para apoio ao manejo sustentável da Floresta A mazônica: possível caminho metodológico. 2003. Dissertação (M estrado em Ciência da Informação) - IBICT, UFRJ, Rio de Janeiro, 2003.

FIGU EIRA NETTO, Silvino C. A comunicação cientifica através de rede de computadores; a experiência de pesquisadores brasileiros. 1994. Dissertação (M estrado em Ciência da Informação) - IBICT, U FRJ, Rio de Janeiro, 1994.

GOMES, Sandra Lúcia Rebel. Bibliotecas virtuais: informação e comunicação para a pesquisa científica. 2002. Tese (D outorado em Ciência da Informação) - IBICT, UFRJ, Rio de Janeiro, 2002.

GRESHAM JUNIOR, John L. From invisible college to cyberspace college: computer conferencing and the transformation of information scholarly communication networks. Interpersonal Computing and Technology, v. 2, n. 4, p .37-52, 1994.

HERT, Philippe. Social dynamics of an on-line scholarly debate. Information Society, London, v. 13, n. 4, p. 329-360, 1997.

MACED O, Tônia M arta Barbosa. Redes(cobrindo) o informal: a inteligência coletiva distribuída. M onografia (Especialização em Inteligência Competitiva) - IBICT, U FRJ, Rio de Janeiro, 1999.

MEADOWS, A. J. A comunicação científica Brasília : Briquet de Lemos Livros, 1999. $268 p$.
Communication in science. London : Butteworths, 1974. 248

p.

; BU CKLE, P. Changing communication activities in the British scientific communication. Journal of Documentation, v. 48, n. 3 , p. 276-290, Sept. 1992.

MERTA, A. Informal communication in science. FID Publications, $\mathrm{n}$. 478, p.34-52, 1972.

PIMIENTA, Daniel; LIENDO, Pablo. La comunicación mediante computadora : una esperanza para los científicos y académicos de América Latina. In: U na nueva manera de comunicar el conocimiento. Caracas: Cresalc, 1993. p.73-100.

PINHEIRO, Lena Vânia Ribeiro. Infra-estrutura da pesquisa em Ciência da Informação. DataG ramaZero, Rio de Janeiro, v. 1, n. 6, dez. 2000. Disponível em: <http:// www.dgz.org.br>

(C ood.). I mpactos das redes eletrônicas na comunicação científica enovos territórios cognitivos para práticas coletivas, interativas eintedisciplinares: relatório final de projeto integrado de pesquisa, 2000-2002. Rio de Janeiro : IBICT, 2002, 80 p.

PEREIR A, M aria de Nazaré F.; PIN HEIRO, Lena Vania R. ( 0 rg.). 0 sonho de 0 tlet: aventura em tecnoloiga da informação e comunicação. Brasília: IBICT, DEP, 2000. 291 p. (Coletânea Projeto Ziman - conhecimento público).

PRICE, Derek de Solla. 0 desenvolvimento da ciência. Trad. S. M athias e G. Braga. Rio de Janeiro : Livros Técnicos e Científicos, 1976. 96 p.

SCHINDT, M iguel Angel.C ontribuição da tecnologia da informação para as geociências-arquitetura de biblioteca virtual de geociências. 2003. Dissertação (M estrado em Ciência da Informação) - IBICT, U FRJ, Rio de Janeiro, 2003.

SCHWEIG, Eugene et al. The Internet: shaking up scientific communication, how a regional mailing list facilitated response to an international event. Nature, July 2001.

TAKAHASHI, Tadao. A Rede Nacional de Pesquisa (RN P): uma visão geral. In: CONGRESSO REGIONAL DE INFORMACC̃̃O EM CIÊNCIAS DA SAÚ DE, 1, 1992, São Paulo. São Paulo : BIREM E, 1992. p. 1-7

VICKERY, Brian. A century of scientific and technical information. Journal of Documentation, v. 55, n. 5, p. 476-527, Dec. 1999.

VIEIRA, Job Lúcio Gomes. Correio eletrônico: o impacto na comunicação técnico científica da EM BRA PA - estudo de caso. 1998. Dissertação (M estrado em Ciência da Informação) - IBICT, U FRJ, Rio de Janeiro, 1998. 